\title{
Transposition
}

Musique et Sciences Sociales

Articles | 2021

\section{L'idéal qui donne sa lumière au silence : Antonio Gramsci et la culture musicale italienne de l'après- guerre}

The Ideal That Illuminates This Silence: Antonio Gramsci and Post-War Italian

Musical Culture

Pietro Milli

\section{OpenEdition}

\section{Journals}

Édition électronique

URL : https://journals.openedition.org/transposition/6629

DOI : 10.4000/transposition.6629

ISSN : 2110-6134

Éditeur

CRAL - Centre de recherche sur les arts et le langage

Référence électronique

Pietro Milli, «L'idéal qui donne sa lumière au silence : Antonio Gramsci et la culture musicale italienne de l'après-guerre », Transposition [En ligne], Articles, mis en ligne le 21 septembre 2021, consulté le 23 septembre 2021. URL : http://journals.openedition.org/transposition/6629 ; DOI : https://doi.org/ 10.4000/transposition.6629

Ce document a été généré automatiquement le 23 septembre 2021.

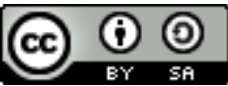

La revue Transposition est mise à disposition selon les termes de la Licence Creative Commons Attribution - Partage dans les Mêmes Conditions 4.0 International. 


\section{L'idéal qui donne sa lumière au silence : Antonio Gramsci et la culture musicale italienne de l'après-guerre}

The Ideal That Illuminates This Silence: Antonio Gramsci and Post-War Italian Musical Culture

Pietro Milli

\section{NOTE DE L'AUTEUR}

Je tiens à remercier vivement le comité de lecture de Transposition, ainsi que les deux évaluateurs anonymes de cet article, pour les précieux conseils qu'ils m'ont prodigués. Mes remerciements vont notamment à Benoît Haug, qui m'a fourni une aide essentielle tout au long de la phase de révision de ce texte.

« l'idéal qui donne sa lumière (mais non pour nous : car tu es mort, et nous sommes morts, avec toi, en ce jardin mouillé) au silence»

- Pier Paolo Pasolini, Les Cendres de Gramsci ${ }^{1}$

\section{Introduction}

1 En 1953, Giacomo Manzoni, alors étudiant en composition au Conservatoire de Milan, recueille des signatures pour une pétition en faveur du couple Julius et Ethel Rosenberg, accusé d'espionnage aux États-Unis ${ }^{2}$, ce qui lui vaut une exclusion de quinze jours. Deux ans plus tard, il adresse une lettre au directeur du quotidien L'Unità, fondé en 1921 par Antonio Gramsci³. Dans cette lettre, il réclame un enseignement plus 
ambitieux de l'éducation musicale dans les écoles, prône une diffusion de la musique dans toutes les strates de la société et incite les compositeurs à se rapprocher de l'«âme populaire ». C'est ainsi que l'on pourra, selon lui, « échapper à l'air putride et irrespirable qui circule dans les tortueux labyrinthes, où se perdent souvent les forces les plus spontanées et les plus engagées des jeunes générations de musiciens ${ }^{4} »$. En 1959, devenu entre-temps critique musical du journal auquel il a adressé cette lettre ${ }^{5}$, le compositeur en herbe traduit Philosophie de la nouvelle musique et Dissonances de Theodor W. Adorno $0^{6}$ : pour la première fois, ces deux œuvres du philosophe francfortois deviennent accessibles dans une langue autre que l'allemand.

Si l'héritage adornien chez les compositeurs de la néo-avant-garde italienne ${ }^{7}$ est largement documenté ${ }^{8}$, l'influence de la pensée de Gramsci sur la culture musicale de cette époque mérite que l'on s'y attarde. Les travaux consacrés à ce sujet ont suivi deux approches différentes. D'une part, on s'est penché sur l'étude de tel ou tel compositeur afin d'appréhender son interprétation de l'œuvre de Gramsci. C'est le cas de l'ouvrage de Joachim Noller traitant de l'engagement musical de Manzoni ${ }^{9}$, et d'un article de Robert Adlington qui analyse Voci destroying muros (1970) de Luigi Nono à la lumière des débats autour de Gramsci dans l'Italie des années 1960-1970 ${ }^{10}$. D'autre part, on a abordé de manière plus générale le retentissement de quelques concepts-clés de la pensée gramscienne (intellectuel organique, national-populaire...) sur la culture musicale italienne du second après-guerre. Par exemple, Luigi Pestalozza a souligné que les réflexions de Gramsci sur la langue ont eu un impact sur toute la musique italienne d'avant-garde depuis les années $1950^{11}$. Gianmario Borio, de son côté, a étudié le projet d'une culture antagoniste mené par le Parti communiste italien (PCI), évoquant à la fois le rock, le jazz et le théâtre d'avant-garde ${ }^{12}$. Une troisième approche, que nous adopterons dans cet article, consiste à s'intéresser à un groupe social plus circonscrit, afin de montrer en quoi les discours et les pratiques de certains acteurs s'inscrivent dans le sillage de Gramsci et relèvent d'un même projet politique. Si cette démarche a déjà été esquissée ${ }^{13}$, nous tenterons de l'entreprendre de manière plus aboutie.

3 Ainsi, nous porterons notre regard sur un groupe de cinq compositeurs, dont la diversité des parcours pourrait faire oublier qu'ils ont partagé à bien des égards un horizon philosophique et esthétique commun: Bruno Maderna, Luigi Nono, Luciano Berio, Sylvano Bussotti et Giacomo Manzoni ${ }^{14}$. Tous ces compositeurs ont considéré Luigi Dallapiccola comme un point de repère artistique et humain incontournable ${ }^{15}$, ont participé aux Cours d'été de Darmstadt dans les années $1950^{16}$ et ont été liés par des rapports amicaux à un moment ou à un autre de leur vie. Parmi eux, Nono est celui qui s'est le plus investi dans les instances politiques, en s'inscrivant au Parti communiste italien en 1952, et en se présentant l'année suivante aux élections parlementaires ; puis, en 1975, en intégrant le Comité central du parti.

Nous tenterons notamment de saisir quelques spécificités dans leur rapport à l'œuvre de Gramsci, en mettant au jour des analogies entre leurs écrits, leurs œuvres et leurs parcours. Mais cette tâche doit faire face à un obstacle majeur : la culture italienne a été si profondément imprégnée de l'héritage gramscien qu'il est difficile d'en saisir la portée, comme l'a souligné Joachim Noller: "L'une des conséquences de l'omniprésence culturelle de Gramsci en Italie est que son nom n'est parfois même pas prononcé ${ }^{17} »$. Par ailleurs, il est important de souligner que la pensée de Gramsci fragmentaire, labyrinthique et parfois insaisissable - ne peut être réduite à un système clos qu'au prix de déformations. Par conséquent, plutôt que de ressasser des formules 
galvaudées visant à synthétiser sa pensée ${ }^{18}$, nous essaierons de mettre en évidence des problématiques nodales qui ressortent de ses écrits et qui se structurent, dans bien des cas, autour de couples de termes opposés. Aussi aborderons-nous dans un premier temps des questions de nature philosophique et sociologique (théorie/praxis, superstructure/infrastructure, intellectuel organique/intellectuel traditionnel, haute culture/culture populaire), et réfléchirons ensuite à des problématiques d'ordre linguistique (la "question de la langue", la dialectique cosmopolitisme/nationalpopulaire et la musique comme langage universel).

Suivant une approche déjà utilisée pour l'étude de la réception d'Adorno ${ }^{19}$, nous mettrons donc au jour certains moments de la pensée de Gramsci et tenterons de saisir leur résonance chez les compositeurs dont il est question. À cet égard, il faut souligner que l'esthétique de ces compositeurs est certes redevable de la pensée de l'intellectuel sarde, mais aussi d'autres philosophes, dramaturges et écrivains ${ }^{20}$, comme Jean-Paul Sartre, Bertolt Brecht et Adorno $^{21}$. L'œuvre de ce dernier divisera même les compositeurs italiens de la néo-avant-garde en deux camps. Nono et Berio $s^{\prime}$ exprimeront de manière très critique à propos de ses analyses ${ }^{22}$, alors que d'autres compositeurs, comme Bussotti, s'approprieront son négativisme et sa conception de l'informel ${ }^{23}$. S'il est illusoire de démêler toutes ces influences, nous croyons qu'il est possible de montrer en quoi ces compositeurs ont appris à "penser de manière gramscienne $»^{24}$, c'est-à-dire à s'approprier les problématiques qu'il a dégagées à son époque pour tenter de répondre aux défis du présent. Cependant, nous n'irons pas jusqu'à juger si ces compositeurs ont été plus ou moins fidèles à la pensée de Gramsci ${ }^{25}$, nous limitant, plus modestement, à exposer leurs prises de position et à s'intéresser à l'inscription de leurs œuvres dans son sillage ${ }^{26}$.

6 À partir de 1947, la maison d'édition Einaudi, à l'initiative du secrétaire du Parti communiste italien Palmiro Togliatti ${ }^{27}$, publie les Lettres de prison $^{28}$, puis les six volumes des Cahiers de prison ${ }^{29}$. Le succès que rencontrent ces écrits est attesté à la fois par l'attribution d'un prestigieux prix littéraire aux Lettres de prison (Premio Viareggio), et par la publication, étalée sur plusieurs décennies, de nombreuses anthologies et d'autres éditions critiques $^{30}$. Dès le départ, la réception de Gramsci en Italie a été conditionnée par la lecture qu'en a donnée Togliatti, comme le rappellent Paolo Capuzzo et Sandro Mezzadra :

Le Parti (notamment sa direction réunie autour de son secrétaire Palmiro Togliatti) s'est approprié Gramsci peu après la fin de la guerre, et sa lecture (ainsi que le travail éditorial autour de ses œuvres inédites) est devenue une pierre angulaire dans la construction d'une " continuité imaginaire » de l'histoire et de la politique du parti depuis sa fondation en $1921^{31}$.

7 Pour Togliatti, Gramsci représentait à la fois une victime du fascisme, à l'instar de Giacomo Matteotti, et une figure emblématique de l'histoire italienne, comme Giordano Bruno, Galileo Galilei ou Giuseppe Garibaldi. On ne peut donc s'étonner qu'une littérature hagiographique se soit développée autour de Gramsci, notamment entre 1945 et $1956^{32}$.

8 S'agissant des compositeurs qui nous intéressent, ils découvrent les écrits de Gramsci entre la fin des années 1940 et le début des années $1950^{33}$. Manzoni, par exemple, a lu les auteurs marxistes à l'époque de ses études au Conservatoire, c'est-à-dire au début des années 1950 : «C'est justement dans ces années que j'ai lu une quantité d'écrits de Marx, de Lénine, d'Engels et de Gramsci, qui a été édité à cette époque par Einaudi. Ce fut un rapprochement progressif, qui a coïncidé, quelques années plus tard, avec mon 
activité de critique musical à $L^{\prime} U n i t \grave{a}^{34}$." Quant à Nono, il aurait connu l'œuvre du philosophe sarde plus tôt: «En 1945, affirme-t-il, je lis les travaux du fondateur du Parti Communiste Italien, Antonio Gramsci. À partir de là, la présence idéologique de Marx est mon point de départ. La lutte contre le fascisme et l'impérialisme est ma raison de vivre. Ce n'est que par hasard que je suis musicien ${ }^{35}$. " Pour la majorité des intellectuels de gauche, la découverte de ces écrits signifiait qu'il était « devenu urgent, une fois encore, de former une nouvelle culture qui puisse donner une véritable expression à l'esprit national, tout en mettant en scène les classes sociales qui en étaient auparavant exclues en tant que protagonistes ${ }^{36}$ ». L'anthropologue Ernesto De Martino $^{37}$ fut l'un des premiers à s'approprier sa pensée afin d'étudier le folklore italien, contribuant ainsi à attirer l'attention sur le monde paysan ${ }^{38}$. Par la suite, Diego Carpitella et Roberto Leydi, profondément influencés par De Martino, compteront parmi les fondateurs de l'ethnomusicologie italienne, tout en côtoyant les compositeurs dont il est question. Leydi, professeur d'ethnomusicologie à l'Université de Bologne et auteur d'importants travaux sur la musique populaire italienne, collabora notamment avec Maderna, Berio et Nono dans le cadre du Studio de phonologie musicale ${ }^{39}$ et de la revue Incontri musicali ${ }^{40}$.

\section{Être compositeur avec Gramsci}

Un des moments les plus féconds de la pensée de Gramsci est constitué sans doute par sa philosophie de la praxis ${ }^{41}$, fondée, entre autres, sur la reformulation du concept de matière. Critique envers les interprétations mécanicistes du matérialisme vulgaire, mais aussi envers l'actualisme de Giovanni Gentile et la dialectique des distincts de Benedetto $\mathrm{Croce}^{42}$, le philosophe sarde souligne que la matière ne doit pas être considérée comme une donnée brute, selon l'exemple des sciences naturelles, ni comme un postulat métaphysique. La matière dépend de l'histoire, car elle est "socialement et historiquement organisée pour la production ${ }^{43}$ ".

Dans sa célèbre conférence "Présence historique dans la musique d'aujourd'hui", Nono souligne précisément l'importance de la catégorie historique d'un point de vue musical, critiquant notamment une certaine utilisation de l'aléa et du collage ${ }^{44}$. Puis il déclare son opposition à toute forme de réductionnisme matérialiste ou idéaliste :

Tantôt c'est l'esprit face auquel la matière n'a que le devoir servile de le refléter fidèlement [...], tantôt c'est la matière à laquelle on attribue des possibilités expressives en soi, alors que, face à ses manifestations autonomes, l'esprit reste passivement en adoration. [...] En réalité, il ne s'agit pas de choisir entre ces deux possibilités [...], parce qu'il n'y a qu'une seule unique possibilité : la connaissance consciente et responsable de la matière par l'intermédiaire de l'esprit, la reconnaissance de la matière atteinte par une réciproque compénétration ${ }^{45}$.

11 Si matière et histoire ne peuvent être appréhendées que dans leur relation réciproque, si l'acte, quelle que soit sa nature, n'est pas totalement déterminé a priori, il s'ensuit que le rapport entre théorie et praxis doit être $\operatorname{revu}^{46}$ : la théorie n'est pas un moment de réflexion précédant la praxis et, à l'inverse, la praxis ne consiste pas en l'application d'une théorie préconçue ${ }^{47}$. À ce propos, Nono ajoute que «la collaboration, la lutte commune avec les travailleurs, le moment idéologique, le moment révolutionnaire et le moment pratique doivent coïncider ${ }^{48} »$. Le compositeur vénitien s'inscrit donc dans le sillage de la philosophie de la praxis gramscienne car il conçoit l'activité compositionnelle comme une théorie/pratique "antagoniste», fondée sur 
l'exploration des possibilités du matériau musical et allant de pair avec l'analyse de son caractère historique. C'est pourquoi Nono, récusant la scission entre culture et politique, dont Croce prônait la nécessité ${ }^{49}$, défend l'idéal d'un art qui se confronte aux problèmes de la cité.

L'opposition théorie/pratique apparait aussi chez Berio, notamment dans sa "Méditation sur un cheval de douze sons $"^{50}$, consacrée à la musique dodécaphonique. Selon Berio, "la démarche du compositeur implique toujours une expérience théorique, mais il est, pourrait-on dire, condamné par la nature même de ses responsabilités à ne jamais réussir pleinement la réconciliation entre théorie et pratique $^{51}$ », puisque l'application d'une procédure préconstituée ne relève pas, selon lui, d'une démarche créative. Ces propos de Berio font écho à ce que Bussotti a affirmé quelques années plus tôt, de manière quelque peu provocatrice : «Il est bon de croire dans les grands révolutionnaires, il y en a qui ont réussi à l'être toute leur vie, sans passer par l'enseignement d'un Conservatoire contre lequel ils avaient jeté auparavant leurs encriers, par exemple Beethoven, Sade, Gramsci sont les morts sur la brèche ${ }^{52}$. " Soulignons aussi que Nono, ainsi que Manzoni, Berio et Bussotti, ont certes produit un nombre considérable d'écrits que l'on peut qualifier de théoriques, mais qu'ils n'ont pas rédigé d'ouvrages comparables à Penser la musique aujourd'hui de Boulez ${ }^{53}$, qui visait, selon Michel Foucault, à «penser la pratique au plus près de ses nécessités internes sans se plier [...] à aucunes d'elles ${ }^{54} »$.

13 Avec sa révision du matérialisme historique, Gramsci propose également une nouvelle interprétation des relations entre superstructure et infrastructure ${ }^{55}$. Si pour Marx et Engels la superstructure est déterminée « en dernière instance » par sa base, Gramsci défend l'idée que toute forme de superstructure agit aussi sur l'infrastructure. Selon lui, les systèmes philosophiques, les religions et les arts ne sont pas le simple reflet d'une base économique, selon une vulgate réductrice marxiste, que Berio critique dans ses écrits :

Entre les différents modes ou «systèmes " (je parle vraiment de poétiques) et la configuration idéologique de la société, il y a toujours une relation étroite. Cette relation n'est pas simple et déterministe, comme certains types de pensée pseudomarxiste voudraient le suggérer (« une société décadente produit une musique décadente $»$ ), mais elle est plutôt dialectique ${ }^{56}$.

14 Cette conception explique, du moins en partie, l'appropriation des moyens technologiques les plus avancés par les compositeurs de la néo-avant-garde italienne. À ce sujet, les propos de Nono sont limpides :

Je suis d'accord avec Antonio Gramsci. Le compositeur doit utiliser les derniers moyens techniques non pas pour des réflexions purement esthétiques, ni au service d'une évolution technologique abstraite, ni passivement, mais activement: pour diffuser les idées dont dépend la lutte des classes. Bien sûr, les rapports de production forment et fixent les conditions préalables à l'art. Mais la superstructure, dont relève le domaine de l'art, peut aussi avoir une fonction dans une relation dialectique à la base. On le voit aujourd'hui dans les mouvements étudiants du monde entier : la base est influencée par la superstructure ${ }^{57}$.

L'utilisation de supports électroniques ${ }^{58}$ peut signifier, par exemple, la volonté de mettre à jour les moyens expressifs pour les retourner contre le système qui les a produits, ainsi que le montrent Atomtod (1964) ${ }^{59}$, œuvre scénique de Manzoni, ou La fabbrica illuminata (1964) de Nono, pour soprano et bande magnétique ${ }^{60}$. L'acte compositionnel n'est donc pas conçu comme un exercice solitaire, mais comme un moment de communication qui peut contribuer à l'évolution de la société. Le 
compositeur, lorsqu'il se confronte à des problèmes linguistiques, participe en effet à la lutte pour l'hégémonie, qui oppose la classe dominée à la classe dominante.

Dans ce cadre, le rôle des institutions devient central : ainsi, de nombreux compositeurs ont plaidé pour une nouvelle organisation de l'enseignement de l'éducation musicale à l'école et au Conservatoire, pour une plus grande ouverture des théâtres et des festivals de musique (comme La Scala et la Biennale de Venise) aux classes sociales subalternes, voire pour la diffusion de la musique savante à la télévision ${ }^{61}$ ou dans les usines, par le biais de concerts et de débats. À ce sujet, il est intéressant de lire le témoignage de Manzoni, qui illustre bien les intentions d'au moins une partie de ces compositeurs :

Souvent, et notamment dans les années 1960 et 1970, à l'initiative de musiciens ou de diverses organisations (surtout des partis de gauche, des associations ou des syndicats), des concerts furent organisés dans des lieux inhabituels, dans des usines, sous des tentes, lors de rassemblements syndicaux et lors des festivals de L'Unità. Lors de ces manifestations, le public «vierge» (pour ainsi dire) pouvait entendre de grands interprètes internationaux, et même des œuvres de compositeurs vivants, et il se montrait sincèrement intéressé et prêt à participer ; c'était une façon concrète de faire comprendre aux personnes exclues d'une si grande partie de la culture [sic] de quelle manière cette musique constituait un patrimoine fondamental, un droit véritable et inaliénable pour tous, dont la réalisation universelle exigeait de nous tous de mener une bataille de civilisation, visant à une transformation radicale des structures en vigueur dans notre pays ${ }^{62}$.

La fondation du Studio de phonologie musicale ou de revues comme Il Diapason ${ }^{63}$ et Incontri musicali peut signifier aussi la volonté d'institutionnaliser des pratiques qui contribuent au renouveau culturel et s'opposent en même temps à toute forme de conservatisme.

Dans cette perspective, le rôle du compositeur ne se limite pas à la production d'œuvres d'art. Si Gramsci affirme que tout être humain, dans une certaine mesure, est un intellectuel, il introduit aussi la distinction entre intellectuels traditionnels et organiques. Les premiers, incarnés entre autres par certains hommes de lettres, philosophes ou artistes, se considèrent comme les «vrais » intellectuels en vertu des fonctions qu'ils occupent, alors que les seconds ne se bornent pas à l'exercice d'une activité spécialisée et assurent le lien avec les masses dans une optique progressiste ${ }^{64}$. Le type d'intellectuel que Gramsci appelle de ses vœux doit en somme "se mêler activement à la vie pratique, comme un constructeur, un organisateur, un "persuadeur permanent" 65 ".

19 Les parcours des compositeurs qui nous intéressent permettent de montrer en quoi ils ont endossé ce rôle ${ }^{66}$ : comme nous l'avons déjà évoqué, Nono s'est engagé de manière très active au sein du $\mathrm{PCI}$, ce qui l'a conduit à se présenter aux élections du Parlement italien ; Berio a été le fondateur d'importantes institutions et revues, alors que Bussotti a été directeur artistique du Teatro la Fenice de Venise, du Festival Pucciniano de Torre del Lago et de la section Musique de la Biennale de Venise ; Manzoni, quant à lui, a été critique musical pour L'Unità et traducteur des œuvres d'Adorno et de Schoenberg. Concernant Maderna, ces quelques lignes écrites par Berio en son hommage sont très éloquentes :

Être compositeur n'était que l'un des nombreux visages, certes le plus important, de sa vie de musicien. [...] Sa participation à la musique a toujours été celle d'un homme entier et complet. Entre ses sens, ses émotions, son intellect, son intuition et sa discipline, il y avait cette unité très précieuse et profonde qui, d'une manière ou d'une autre, représente depuis toujours le thème universel de la musique : c'est- 
à-dire celui qui consiste à trouver et à perfectionner la compréhension entre différentes dimensions, entre dire et faire, penser et agir, entre « corps et âme ${ }^{67}$. répertoire des musiques diffusées à large échelle par les médias et, d'autre part, par les musiques issues de traditions locales. Afin de s'approprier ces deux formes de musique populaire, les compositeurs de la néo-avant-garde italienne ont investi différentes trajectoires. Par exemple, dans leurs œuvres apparaissent parfois des citations, plus ou moins reconnaissables, enrichissant le matériau musical par une mise en perspective historique, comme dans Venetian Journal (1972) de Maderna, pour ténor orchestre et bande magnétique ${ }^{72}$, et dans Al gran sole carico d'amore (1974) de Nono, action scénique en deux parties ${ }^{73}$. Les Folk Songs (1964) de Berio, pour mezzo-soprano et sept instrumentistes, relèvent quant à elles d'un travail de recomposition, dans lequel le discours instrumental doit «suggérer et commenter [...] les racines expressives ${ }^{74}$ » de chansons issues de différentes cultures (française, arménienne, sicilienne...). Le même compositeur a aussi arrangé des chansons rock dans Beatles songs (1967), pour voix et ensemble, alors que dans Coro (1974-1976), pour quarante voix et instruments, il a utilisé «des modes populaires et des techniques les plus diversifiés, sans aucune référence à des chants spécifiques ${ }^{75}$." Manzoni, qui a participé au groupe Cantacronache dans les années $1950^{76}$, s'est inspiré, comme Berio, des musiques populaires siciliennes, notamment dans Cinque vicariote (1958), pour chœur mixte et orchestre $^{77}$. De son côté, Nono a utilisé des textes d'ouvriers et des sons concrets enregistrés en usine (La fabbrica illuminata, 1964, pour soprano et bande magnétique), ainsi que des graffitis et slogans de mai 1968 (Musica-Manifesto n. 2: Non consumiamo Marx, 1969, pour bande magnétique), adoptant ainsi une démarche nettement différente de celles de ses contemporains. 


\section{La « quistione della lingua » et ses enjeux musicaux et politiques}

24 La question de la culture populaire soulève en même temps la "quistione della lingua ", à la fois du point de vue musical et littéraire. Comme le rappelle Gramsci :

Chaque fois qu'affleure, d'une façon ou d'une autre, la question de la langue, cela signifie qu'une série d'autres problèmes est en train de s'imposer : la formation et l'élargissement de la classe dirigeante, la nécessité d'établir des rapports plus intimes et plus sûrs entre les groupes dirigeants et la masse populaire-nationale, c'est-à-dire de réorganiser l'hégémonie culturelle ${ }^{78}$.

Sur le plan musical, cela implique que le langage musical utilisé par un compositeur révèle à la fois son esthétique et sa position - dans le cadre d'une "guerre de positions $»^{79}$ - dans la lutte pour l'hégémonie culturelle ${ }^{80}$. Aussi Pestalozza souligne-t-il que le Studio de phonologie musicale, se concentrant sur l'élaboration d'un matériau acoustique instrumental et vocal, a pris ses distances avec l'école parisienne du Groupe de recherches musicales et du Studio de la radio de Cologne, où l'aspect technologique a selon lui prédominé ${ }^{81}$. Sur le plan littéraire, les caractéristiques linguistiques du texte à mettre en musique ont elles aussi été matière à réflexion. Par exemple, l'utilisation de plusieurs langues au sein d'une même œuvre a permis à nombre de compositeurs non seulement d'enrichir les possibilités expressives du matériau musical d'un point de vue phonétique, mais aussi de souligner le caractère universel des thématiques abordées ${ }^{82}$. Rappelons aussi que certains compositeurs ont mis en musique des textes écrits dans des idiomes régionaux, dont l'utilisation n'est pas fortuite ${ }^{83}$.

Si l'utilisation d'une langue dans une œuvre musicale soulève des enjeux qui vont audelà du cadre purement esthétique, les concepts gramsciens de cosmopolitisme et de national-populaire peuvent aider à en préciser les contours. Dans ses écrits, Gramsci constate à la fois l'absence, en Italie, d'une véritable culture et littérature nationalepopulaire et le caractère cosmopolite de ses intellectuels. Cosmopolitisme et nationalpopulaire peuvent tout de même être l'expression du même phénomène. En prenant l'exemple de Benedetto Croce, qu'il considère comme le dernier homme de la Renaissance, Gramsci affirme qu'il a cherché à élever la culture italienne, trop provinciale, au niveau international, tout en poursuivant une finalité essentiellement nationale par son attitude et par sa fonction au sein de la société.

Dans un entretien avec José Antonio Alcaraz, Nono aborde précisément cette problématique. Interrogé à propos de ses préférences concernant les compositeurs italiens d'opéras, il répond que Bellini, Verdi, Busoni et Dallapiccola ont été pour lui des points de repère fondamentaux, avant de préciser: «L'enseignement génial de ces musiciens s'intensifie s'il s'intègre à d'autres conceptions théâtrales européennes, mais aussi plus lointaines en apparence, comme celles des théâtres japonais (kabuki et nô) et chinois ${ }^{84}$. » Après avoir insisté sur la nécessité de connaître non seulement la tradition musicale européenne, mais aussi les musiques du monde, Nono ajoute :

Nationalisme ou cosmopolitisme? Une telle dichotomie tient d'un schématisme superficiel, semblable à celui de la forme et du contenu. L'intelligence et la capacité humaine de penser et d'inventer annulent ces manifestations trop étroites et catégorielles ${ }^{85}$.

Aussi n'est-il pas surprenant que dans Doktor Faustus (1988), œuvre scénique inspirée du roman éponyme de Thomas Mann, Manzoni s'approprie un mythe appartenant à une 
culture étrangère par le biais de la langue. En effet, le compositeur a choisi de mettre en musique un livret constitué de citations du roman mannien traduites en italien, expliquant ainsi les raisons qui l'ont poussé à ne pas utiliser le texte original allemand :

Plus l'auteur du roman, et donc son propre protagoniste, étaient immergés dans la culture allemande, dans son histoire, dans ses mythes et dans ses tragédies, moins il devait sembler possible de s'identifier à eux en partant d'une langue et d'une histoire aussi différentes que celles de l'Italie par rapport à celles de l'Allemagne (mais à l'intérieur d'une grande unité culturelle européenne). S'affirme alors [...] la nécessité de se distinguer, de se poser comme autre au moment même de la confrontation, de ne pas se chercher une identité étrangère, qui n'aurait pu mener qu'à l'échec. Ainsi lui, le musicien, doit prendre pour ainsi dire ses distances : plus l'échec serait sensationnel, précisément, plus convaincante serait l'éventuelle capacité à saisir le noyau universel du Faustus mannien, de manière autonome, audelà des distinctions de langue, de culture et d'histoire ${ }^{86}$.

D'autres démarches existent à côté de celle-ci : dans Satyricon (1973) de Maderna, par exemple, c'est l'évocation d'un lieu (la Rome antique décrite par Pétrone) qui symbolise la rencontre des cultures et des peuples les plus variés, mise en évidence ici aussi par le foisonnement des langues utilisées. Sur un autre versant, l'approche utilisée par Stockhausen dans Hymnen (1966-1967), musique électronique et concrète mêlant différents hymnes nationaux, mais pas seulement, a été durement critiquée à la fois par Pestalozza $^{87}$ et par Nono. Selon ce dernier, Stockhausen incarnerait «la théorisation d'une évolution technologico-esthétique indolore, la relation naturelle avec les lieux de la production technique la plus avancée, c'est-à-dire les États-Unis et l'Occident, et le mépris aristocratique pour toutes les autres cultures ${ }^{88}$. » Plus particulièrement, Nono y décèle la présence d'un "sentiment de supériorité 89 ", qui ressortirait du choix et de l'élaboration du matériau musical.

Selon Gramsci, la musique elle-même n'échappe pas à la dialectique que nous avons évoquée. Comme l'intellectuel sarde le souligne, elle est certes le langage universel par excellence, mais elle se fonde sur une "substance culturelle " qui est de nature nationale-populaire: une œuvre de Verdi, par exemple, peut être appréciée par n'importe quel citoyen du monde, mais elle ne sera pas pour autant perçue de la même manière par un italien ou par un japonais. Se penchant sur la situation de son pays, Gramsci constate également qu' « en Italie, la musique a, dans une certaine mesure, remplacé dans la culture populaire l'expression artistique qui, dans d'autres pays, est fournie par le roman populaire ${ }^{90}$ ". Il remarque alors que les livrets d'opéras italiens ont souvent un caractère européen, que l'on peut déceler dans l'intrigue du drame et dans le type de sensibilité qui en ressort, et qu' " au moment où, dans chaque pays, se produit une stricte nationalisation des intellectuels indigènes [...], les intellectuels italiens poursuivent à travers la musique leur fonction européenne ${ }^{91} »$. De plus, si Gramsci soutient que le mélodrame verdien a rencontré la même fortune auprès des masses populaires que les romans d'Eugène Sue en France (qui relèveraient, quant à eux, d'une littérature commerciale), sa popularité doit plutôt être comparée, selon lui, à celle des tragiques grecs ou de Shakespeare, auteurs qui «sont essentiellement populaires dans tous les pays ${ }^{92} »$.

31 Comme le rappelle Berio, les compositeurs de l'après-guerre ont été profondément influencés par la lecture gramscienne (et plus généralement marxiste) du mélodrame italien : « Après la chute du fascisme, la redécouverte du mélodrame italien à la lumière des lectures de Gramsci et de Lukács, c'est-à-dire à la lumière de la critique marxiste, a été très importante ${ }^{93}$.» Concernant Verdi, par exemple, on peut constater qu'il est 
présent dans l'univers de nombre d'entre eux, que ce soit sous la forme d'une citation musicale (les trompettes d'Aida dans Satyricon de Maderna), d'un emprunt (l'échelle énigmatique utilisée par Nono dans Fragmente-Stille, an Diotima, 1980, pour quatuor à cordes), d'une référence esthétique (La vera storia, 1982, opéra en deux actes de Berio, s'inspirant de Il Trovatore), d'une indication de mise en scène (La Passion selon Sade, 1966, mystère de chambre de Bussotti $\left.{ }^{94}\right)$, ou d'articles ${ }^{95}$. Le répertoire du théatre musical italien de la seconde moitié $\mathrm{du} \mathrm{xx}^{\mathrm{e}}$ siècle fait aussi bien souvent référence à l'expérience tragique, révélant ainsi le souci d'aborder des thématiques allant au-delà de toute frontière, selon l'exemple de la littérature grecque et anglaise, mais aussi de Verdi.

\section{Conclusion}

D'autres questions d'ordre linguistique et politique pourraient être posées au sujet des œuvres de ces compositeurs dont le matériau poétique provient précisément des écrits privés de Gramsci, dès lors que «le choix et le traitement des textes représentent différentes formes d'engagement $»^{96}$. Le degré d'intelligibilité du texte, ainsi que son exploitation musicale, dénotent en effet les intentions et les positions des compositeurs, tantôt de manière explicite, tantôt de manière indirecte. Ce sujet mériterait d'être abordé dans le cadre d'une étude comparative, mais nous nous contenterons ici d'une évocation des quelques œuvres relatives aux Lettres de prison de Gramsci. Publiées avant les Cahiers de prison, dans une version censurée par le Parti ${ }^{97}$, ces lettres ont d'emblée forgé l'image du révolutionnaire antifasciste : «[Elles] ont mis en évidence le côté humain de Gramsci et ont signifié de manière paradigmatique la violence subie par tous ceux qui ont eu le courage de s'opposer vigoureusement au régime fasciste ${ }^{98}$.» Dans le milieu musical, la publication de ces lettres a eu un retentissement important: "Dans une de nos longues conversations [...]», affirme Pestalozza, «il [Nono] me dit que dans les lettres de Gramsci comme dans celles des anti-fascistes et des acteurs de la rupture historique, le privé et le public, l'histoire concrète, ce qui concerne également dans le futur tous les hommes, vont de pair ${ }^{99}$.»

Aussi les lettres de Gramsci révèleraient-elles à la fois la tragédie d'une vie qui se consume dans la lutte pour un idéal et le " rapport mystifié avec les autres hommes ${ }^{100}$ ", régi par la relation dominant/dominé. Un tel entrelacs entre vie publique et vie privée se retrouve en premier lieu dans Vier Briefe. Kranichsteiner Kammerkantate (1953) de Maderna, œuvre pour soprano, basse et ensemble ${ }^{101}$. Dans cette composition, un montage de deux lettres de Gramsci, adressées à sa femme et à sa belle-sœur, côtoie une lettre d'un condamné à mort de la Resistenza, des extraits de lettres commerciales anonymes, ainsi qu'une lettre de Franz Kafka adressée à Milena Jesenská. Par ailleurs, en 1962, Bussotti commence à travailler à I semi di Gramsci (1962-1971), poème symphonique pour quatuor à cordes et orchestre - dont le titre n'est pas sans rappeler un recueil de poèmes de Pier Paolo Pasolini, intitulé Les cendres de Gramsci (1957) - où le musicologue Massimo Mila entend dans le quatuor une incarnation de Gramsci, et dans l'orchestre une représentation de sa prison ${ }^{102}$. Pour cette composition instrumentale, Bussotti utilise comme source d'inspiration des extraits de trois lettres de Gramsci, reproduites en haut de chaque page de la partition, et devant être distribuées au public. D'après une lettre que Bussotti a envoyée à Manzoni, Adorno aurait joué un rôle très important dans l'élaboration de cette partition: "Sans ces conversations inoubliables avec lui [...], je n'aurais jamais été capable de mener à terme la partition des Semi di 
Gramsci. Puisque c'est Adorno qui m'enseigna à nouveau "l'articulation linguistique" au sens musical s'entend - des phrases ${ }^{103}$. »

Si le survol proposé par cet article nous a permis de montrer combien l'œuvre de Gramsci a pénétré la pensée musicale et politique des compositeurs de la néo-avantgarde italienne, deux questions restent ouvertes. Premièrement, on peut se demander quelle utilisation a été faite de Gramsci : s'est-il agi d'une instrumentalisation à des fins d'autolégitimation, comme l'a laissé entendre une partie de la gauche italienne ${ }^{104}$, ou bien d'une appropriation visant à rendre plus cohérent un projet social et culturel ? Manzoni résume ainsi les ambitions de sa génération : "Nous aspirions à une nouvelle société en Italie, distincte de l'URSS, plus attentive aux droits de l'homme, aux nouvelles idées concernant le développement, la recherche et la liberté, dans la musique et dans les arts en général ${ }^{105}$. „ Ce qui est indéniable, c'est que certains de ces compositeurs, s'inscrivant dans le sillage de Gramsci, ont pris leurs distances avec l'idéal de l'art pour l'art, d'une part, et avec le modèle soviétique (l'art comme propagande), d'autre part. Plus généralement, Guido Liguori remarque que «la tradition italienne et la méthode de Gramsci représentaient [...] la recherche d'un terrain de rencontre avec la culture de notre pays ; mais elles ont aussi été la voie et le moyen [...] pour prendre des distances avec le modèle stalino-jdanoviste, sans pour autant déterminer une rupture ouverte et politiquement insoutenable ${ }^{106}$. » Il s'ensuit que l'influence de Gramsci sur la culture italienne de cette époque ne peut pas être étudiée uniquement sur la base des contenus que son œuvre véhicule. La méthode d'analyse de Gramsci, sa façon d'appréhender le réel, a fait partie de son héritage tout aussi bien que l'arsenal de concepts qu'il a élaborés.

Un autre questionnement, non moins important, concerne la manière dont certains de ces compositeurs, et plus généralement nombre d'intellectuels de cette époque, ont assimilé non seulement la pensée de Gramsci mais également celle d'Adorno. À ce sujet, l'exemple de Manzoni et de Bussotti, qui ont tous les deux connus le philosophe francfortois, est emblématique. Or, y a-t-il des points de rencontres possibles entre Gramsci et Adorno ? Domenico Losurdo semble répondre par la négative :

Gramsci n'a pas été seulement un penseur, il a été également un dirigeant communiste de premier plan: il ne peut pas être vu comme une sorte de Horkheimer ou d'Adorno italien engagé dans la construction d'une théorie critique sans rapport ou avec un rapport exclusivement polémique à l'égard du mouvement communiste et du « mouvement réel » de transformation de la société. Le problème de l'unité entre intellectuels et conscience commune est absent de l'école de Francfort. Celle-ci reprend idéalement les positions d'Érasme auquel les Quaderni del carcere (Cahiers de prison) opposent Luther de façon répétitive et positive. Loin de posséder la finesse et la culture du grand humaniste, le grossier réformateur met en route cependant un processus de transformation tumultueuse : il exprime dans sa rusticité le travail de l'accouchement d'une société nouvelle ${ }^{107}$.

Manzoni, quant à lui, récuse certes la conception consumériste de l'art entretenue par l'industrie culturelle, s'inscrivant ainsi dans le sillage d'Adorno, mais il rejette en même temps son solipsisme, prônant la nécessité d'établir une communication avec les masses. Et vingt-cinq ans après la parution de la Théorie esthétique ${ }^{108}$, il appelle de ses vœux non pas une esthétique, mais une philosophie de la musique, dont l'objectif est de "vérifier presque quotidiennement une praxis compositionnelle qui ne doit jamais oublier la responsabilité et le sens de son existence même et de son faire. ${ }^{109}$ " Dès lors, si une conciliation entre Adorno et Gramsci a été possible, c'est sans doute car le premier 
a fourni des outils pour comprendre et critiquer la société de l'après-guerre, alors que le second a fait entrevoir le projet, toujours à accomplir, d'une nouvelle société.

\section{BIBLIOGRAPHIE}

ADORNO Theodor W., Filosofia della musica moderna, Turin, Einaudi, 1959 [1949].

ADORNO Theodor W., Dissonanze, Milan, Feltrinelli, 1959 [1956].

ADORNO Theodor W., Théorie esthétique, Paris, Klincksieck, 1974 [1970].

ADLINGTON Robert, « Whose Voices ? The Fate of Luigi Nono's Voici destroying muros ", Journal of the American Musicological Society, 69/1, 2016, p. 179-236.

ARBO Alessandro, « La musicologia di Adorno nella sua ricezione italiana (1950-1980) », Nuova rivista musicale italiana, $\mathrm{n}^{\circ} 2,1988$, p. 181-210.

ASOR ROSA Alberto, Intellettuali e classe operaia, Florence, La Nuova Italia, 1973.

BERIO Luciano, « Coro (note de l'auteur) » (http://www.lucianoberio.org/node/1435?1048623366=1, consulté le 15 mars 2021).

BERIO Luciano, « Folk Songs (note de l'auteur) », (http://www.lucianoberio.org/node/1380?

37697026=1, consulté le 15 mars 2021).

BERIO Luciano, Entretiens avec Rossana Dalmonte : Écrits choisis, Genève, Éditions Contrechamps, 2010.

вовВіо Norberto, «Il nostro genio speculativo », Il Contemporaneo, 1955, n² 24, p. 1-2.

BERIO Luciano, Scritti sulla musica, Turin, Einaudi, 2013.

BORIO Gianmario, « Key Questions of Antagonist Music-Making: A View from Italy », ADLINGTON Robert (dir.), Red Strains: Music and Communism outside the Communist Bloc, Londres, British Academy, 2013, p. 175-191.

BOULEz Pierre, Penser la musique aujourd'hui, Paris, Denoël/Gonthier, 1987 [1964].

CAPUZZo Paolo et MEZZADRA Sandro, « Provincializing the Italian Reading of Gramsci », SRIVASTAVA Neelam et BHATTACHARYA Baidik (dir.), The Postcolonial Gramsci, New York, Routledge, 2012, p. 34-54.

CHARUTY Giordana, « Être ensemble dans la même histoire : l'œuvre-vie d'Ernesto De Martino », Bérose - Encyclopédie internationale des histoires de l'anthropologie, Paris, 2018 (en ligne : https:// www.berose.fr/article1428.html, consulté le $1^{\mathrm{er}}$ mars 2021).

DE MARTINO Ernesto, « Intorno a una storia del mondo popolare subalterno », Società, $1949, \mathrm{n}^{\circ} 3$, p. 411-435.

ESPOSITo Luigi, Un male incontenibile. Sylvano Bussotti, artista senza confini, Bietti, Milan, 2013.

FAVARA Antonio, Corpus di musiche popolari siciliane, Palerme, Accademia di scienza, lettere e arti, 1957. 
FENEYROU Laurent, « Musiques informelles, musiques négatives. Sylvano Bussotti, Aldo Clementi, Franco Donatoni, Giacomo Manzoni, Franco Evangelisti », DONIN Nicolas et Laurent FENEYROU (dir.), Théories de la composition musicale au XXe siècle, Lyon, Symétrie, 2013, vol. I, p. 735-776.

FENEYROU Laurent, « ...la révolution ne renie pas la beauté... Musique et marxisme dans l'Italie d'après 1945 ", FENEYROU Laurent (dir.), Résistances et utopies sonores, musique et politique au XXe siècle, Paris, CDMC, 2005, p. 149-180.

FOUCAUlT Michel, « Pierre Boulez ou l'écran traversé », préface posthume à BoulEz Pierre, Leçons de musique : deux décennies d'enseignement au Collège de France (1976-1995), Paris, Christian Bourgois, 2005 [1982], p. 19-22.

FRÉTIGNÉ Jean-Yves, Antonio Gramsci : vivre, c'est résister, Paris, Armand Colin, 2017.

GRAMSCI Antonio, Lettere dal carcere, Turin, Einaudi, 1947.

GRAMSCI Antonio, Quaderni del carcere, Turin, Einaudi, 1948-1951.

GRAMSCI Antonio, Cahiers de prison, Paris, Gallimard, 1978-1996.

HALL Stuart, « Gramsci and us », Marxism Today, 1987, p. 16-21.

HOARE George et Nathan SPERBER, Introduction à Antonio Gramsci, Paris, La Découverte, 2019 [2013].

LIGUORI Guido, Gramsci conteso : Interpretazioni, dibattiti e polemiche (1922-2012), Rome, Editori Riuniti, 2012.

LOMBARDI Daniele (dir.), Giacomo Manzoni : Pensare attraverso il suono, Milan, Mudima, 2016.

LOSURDo Domenico, « Avec Gramsci, par-delà Marx et par-delà Gramsci », Nouvelles Fondations, 2007/3-4, no 7-8, p. 210-218.

MACCIOcCHI Maria-Antonietta, Pour Gramsci, Paris, Le Seuil, 1974.

MANZONI Giacomo, Scritti, Florence, La Nuova Italia, 1991.

MANZONI Giacomo, « La leçon d'Adorno à l'époque de la néo-avant-garde » [1980], FENEYROU Laurent (dir.), Résistances et utopies sonores, Paris, CDMC, 2005, p. 119-128.

MANZONI Giacomo, Écrits, Paris, Basalte, 2006.

MANZONI Giacomo, Parole per musica, Palerme, L'Epos, 2007.

MANZONI Giacomo, Musica e progetto civile. Scritti e interviste (1956-2007), Milan, Ricordi/LIM, 2009.

MANZONI Giacomo, « Towards Political and Musical Renewal: The Other Idea of Communism », ADLINGTON Robert (dir.), Red Strains: Music and Communism outside the Communist Bloc, Londres, British Academy, 2013, p. 23-29.

MICHEL Pierre, Luigi Dallapiccola, Genève, Éditions Contrechamps, 1996.

MILA Massimo, « Un omaggio musicale all'opera di Gramsci », La Stampa, 28 août 1974 (http:// heinrichvontrotta.blogspot.com/2011/05/sylvano-bussotti-i-semi-di-gramsci.html, consulté le 15 mars 2021).

MILLI Pietro, « Théâtre musical et matiérisme : Atomtod de Giacomo Manzoni », Itamar, $\mathrm{n}^{\circ}$ 5, 2019, p. $187-206$.

MILLI Pietro, « Dialectics and Matter in the Aesthetics of Giacomo Manzoni », Archival Notes, $\mathrm{n}^{\circ} 5$, 2020, p. 47-58. 
NOLLER Joachim, Engagement und Form: Giacomo Manzonis Werk in kulturtheorischen und musikhistorischen Zusammenhängen, Francfort, Peter Lang, 1987.

NOLLER Joachim, « Gramsci e la musica », Musica/Realtà, X, nº 30, décembre 1989, p. 35-48.

NONO Luigi, Texte, Studien zu seiner Musik, Zurich, Atlantis, 1975.

NONO Luigi, Écrits, Genève, Contrechamps, 2007.

PASOLINI Pier Paolo, Les Cendres de Gramsci, Paris, Gallimard, 2017 [1957].

PESTALOZZA Luigi, « Impegno ideologico e tecnologia elettronica nelle opere degli anni Sessanta », RESTAGNo Enzo (dir.), Nono, Turin, EDT, 1987, p. 143-156.

PESTALOZZA Luigi, Musique, rupture, Paris, Éditions Delga, 2012.

QUASIMODO Salvatore, Il Falso e vero verde. Con un discorso sulla poesia, Milan, A. Mondadori, 1956.

SALVETTI Guido, «Ideologie politiche e poetiche musicali nel Novecento italiano », Rivista italiana

di musicologia, XXXV, $\mathrm{n}^{\circ}$ 1/2, 2000, p. 107-133.

SAMUEL Jamuna, « Ethics and Musical Language: A Gramscian Reading of Dallapiccola's Liriche

greche and Their Influence ", Revue canadienne de musique, XXXV, $\mathrm{n}^{\circ}$ 1, 2015, p. 123-153.

TODDE Felice, «Gramsci e la musica », Nuova rivista musicale italiana, XXIX, n 3, 1995, p. 491-500.

TODDE Felice, «L'opera lirica nel pensiero di Antonio Gramsci », Nuova rivista musicale italiana,

XLIII, $\mathrm{n}^{\circ}$ 3, 2009, p. 347-367.

TOGLIATTI Palmiro, Scritti su Gramsci, Rome, Editori Riuniti, 2001.

VERZINA Nicola, « Tecnica della mutazione e tecnica seriale in Vier Briefe (1953) di Bruno

Maderna », Rivista italiana di musicologia, XXXIV, nº 2, 1999, p. 309-346.

\section{NOTES}

1. PASOLINI Pier Paolo, Les Cendres de Gramsci, Paris, Gallimard, 2017 [1957], p. 21.

2. Voir MANZONI Giacomo, «Musica nuova per una nuova società. Colloquio con Giacomo Manzoni di Raffaele Pozzi », Pozzi Raffaele (dir.), Musica e progetto civile. Scritti e interviste (1956-2007), Milan, Ricordi/LIM, 2009, p. 440.

3. Voir MANZONI Giacomo, « Musica e pubblico » [1955], Scritti, Florence, La Nuova Italia, 1991, p. 5-7. Pour une biographie de Gramsci en langue française, voir FRÉTIGNÉ JeanYves, Antonio Gramsci: vivre, c'est résister, Paris, Armand Colin, 2017. Nous tenons également à signaler deux ressources qui peuvent aider à s'orienter dans l'univers gramscien: http://www.gramsciproject.org (archive numérique des textes, des contenus et des applications concernant Gramsci, consultée le 15 mars 2021) ; http:// bg.fondazionegramsci.org/biblio-gramsci (outil de recherche bibliographique des écrits de et sur Gramsci, consulté le 15 mars 2021).

4. " sfuggire all'aria putrida e irrespirabile che gravita in quei tortuosi labirinti nei quali si perdono sovente le forze più genuine e più impegnate delle giovani generazioni di musicisti. » MANZONI, «Musica e pubblico», p. 7. Sauf mention contraire, les traductions depuis l'italien, l'anglais et l'allemand sont de notre fait.

5. Manzoni a été critique musical de L'Unità entre 1958 et 1966. 
6. ADORNo Theodor W., Filosofia della musica moderna, Turin, Einaudi, 1959 [1949]; Dissonanze, Milan, Feltrinelli, 1959 [1956].

7. Cette expression peut être critiquée, comme toute sorte d'étiquette. Par exemple, Luciano Berio affirme : "Néo-avant-garde" est une de ces expressions dont on se sert quand le compte n'y est plus, quand on cherche à isoler un phénomène de façon générale et arbitraire, en essayant de faire passer pour homogène ce qui ne l'est pas ", BERIO Luciano, Entretiens avec Rossana Dalmonte: Écrits choisis, Genève, Éditions Contrechamps, 2010, p. 49 (cette série d'entretiens fut publiée pour la première fois en 1981). Néanmoins, nous utiliserons ce terme car il nous permet d'identifier plus aisément les compositeurs dont il est question ici. Nous ne voulons pas pour autant effacer les différences, parfois abyssales, qui séparent les univers de ces compositeurs.

8. Voir notamment PESTALOZZA Luigi, «La contradiction pratique chez Adorno » [1960], Musique, rupture, Paris, Éditions Delga, 2012, p. 9-26; MANZONI Giacomo, «La leçon d'Adorno à l'époque de la néo-avant-garde » [1980], FENEYRou Laurent (dir.), Résistances et utopies sonores, Paris, CDMC, 2005, p. 119-128; ARBO Alessandro, «La musicologia di Adorno nella sua ricezione italiana (1950-1980) ", Nuova rivista musicale italiana, $\mathrm{n}^{\circ} 2$, 1988, p.181-210; FENEYROU Laurent, "Musiques informelles, musiques négatives. Sylvano Bussotti, Aldo Clementi, Franco Donatoni, Giacomo Manzoni, Franco Evangelisti », DONIN Nicolas et FENEYROU Laurent (dir.), Théories de la composition musicale au XXe siècle, Lyon, Symétrie, 2013, vol. I, p. 735-776.

9. NOLLER Joachim, Engagement und Form: Giacomo Manzonis Werk in kulturtheorischen und musikhistorischen Zusammenhängen, Francfort, Peter Lang, 1987.

10. ADLINGTON Robert, "Whose Voices? The Fate of Luigi Nono's Voci destroying muros", Journal of the American Musicological Society, 69/1, 2016, p. 179-236.

11. PESTAlozZA Luigi, «Gramsci, la langue, la musique : aujourd'hui », Musique, rupture, 2002, p. 27-38. Parmi les compositeurs italiens influencés par Gramsci, Pestalozza évoque notamment Maderna, Nono, Berio, Manzoni, Franco Oppo et Andrea Liberovici.

12. BORIO Gianmario, «Key Questions of Antagonist Music-Making: A View from Italy », ADLINGTON Robert (dir.), Red Strains: Music and Communism outside the Communist Bloc, Londres, British Academy, 2013, p. 175-191.

13. Voir NOLLER Joachim, «Gramsci e la musica », Musica/Realtà, X, n 30, décembre 1989, p. 35-48. Ce court article étudie principalement l'influence de Gramsci chez Luigi Nono, mais il aborde aussi, quoique très rapidement, deux œuvres de Bruno Maderna et de Sylvano Bussotti. Si quelques concepts fondamentaux de la pensée gramscienne ont été mis en évidence par l'auteur (hégémonie, intellectuel, folklore...), on peut regretter le caractère quelque peu décousu de l'article, qui fait par ailleurs abstraction de la réception des Cahiers de prison et des Lettres dans l'Italie de cette époque. Pour une étude plus générale sur les rapports entre musique et politique dans l'Italie du $\mathrm{xx}^{\mathrm{e}}$ siècle, voir SALVETTI Guido, «Ideologie politiche e poetiche musicali nel Novecento italiano », Rivista italiana di musicologia, XXXV, $\mathrm{n}^{\circ} 1 / 2,2000$, p. 107-133. Concernant l'intérêt de Gramsci pour la musique et ses positions sur l'opéra, voir TODDE Felice, "Gramsci e la musica ", Nuova rivista musicale italiana, XXIX, $\mathrm{n}^{\circ} 3$ 3, 1995, p. 491-500 et, du même auteur, « L'opera lirica nel pensiero di Antonio Gramsci ", Nuova rivista musicale italiana, XLIII, $\mathrm{n}^{\circ} 3,2009$, p. 347-367. 
14. Des interprètes de renom ont participé à des initiatives menées par le $\mathrm{PCI}$ et se sont parfois liés d'amitié avec ces compositeurs. Parmi eux, citons le chef d'orchestre Claudio Abbado, les pianistes Bruno Canino et Maurizio Pollini, ainsi que le flûtiste Severino Gazzelloni.

15. Concernant les relations entre Luigi Dallapiccola (1904-1975), d'une part, et Nono, Berio et Bussotti, d'autre part, voir : MICHEL Pierre, Luigi Dallapiccola, Genève, Éditions Contrechamps, 1996, p. 124-135. Voir aussi MANZoNi Giacomo, « Dallapiccola: l'etica della libertà », Scritti, 1975, p. 233-236.

16. Les œuvres de Maderna et Nono ont été mises au programme des Cours d'été de Darmstadt dès 1950 ; suivent celles de Berio (1956), de Manzoni (1957) et de Bussotti (1959).

17. «Gramscis allgegenwärtige kulturelle Präsenz in Italien kann zur Folge haben, dass der Name selbst nicht fällt. » NOLLER Joachim, Engagement und Form: Giacomo Manzonis Werk in kulturtheorischen und musikhistorischen Zusammenhängen, Francfort, Peter Lang, 1987, p. 78.

18. À ce sujet, Norberto Bobbio affirme : «Gramsci a été réduit, y compris par les marxistes italiens, à un inventaire de cinq ou six formules par lesquelles on explique toutes sortes de choses, et ses livres sont devenus une somme de maximes ou versets que l'on cite en tant qu'arguments ex auctoritate » ( di Gramsci si è fatto, i marxisti italiani hanno fatto, un inventario di cinque o sei formule con le quali si spiega ogni cosa, e dei suoi libri una somma di massime o versetti da citare come argomentiex auctoritate »), BOBBIoNorberto, «Il nostro genio speculativo », Il Contemporaneo, 1955, n 24, p. 2.

19. Voir MANZONI, « La leçon d'Adorno... ».

20. Nous n'évoquerons pas ici les influences musicales, qui ont été tout aussi importantes.

21. Comme le rappelle Gianmario Borio, l'un des traits d'union entre ces contributions disparates est la critique de la société capitaliste : «De nombreux courants de la pensée contemporaine qui ont alimenté la critique de la société capitaliste tardive (Herbert Marcuse, Guy Debord, la Beat Generation, les nouvelles approches de la pédagogie et de la psychiatrie) ont coexisté, et dans certains cas se sont mélangés, avec la perspective gramscienne " "Many of the strands of contemporary thought which fed into the critique of late-capitalist society (Herbert Marcuse, Guy Debord, the Beat Generation, and new approches in pedagogy and psychiatry) coexisted, and in certain cases mixed, with the Gramscian perspective"), BORIO, « Key Questions... », p. 178.

22. Selon Nono, Adorno et Horkheimer «s'en remettent à une sociologie nordaméricaine libérale, à laquelle ils se limitent ", NONo Luigi, "Le pouvoir musical» [1969], Écrits, Genève, Contrechamps, 2007, p. 315. Berio, quant à lui, critique à la fois ses analyses sociologiques et sa conception de la musique: "la vision musicale apocalyptique d'Adorno tend à perdre tout contact avec les réalités de notre monde, comme si l'unique et ultime destination de toute œuvre musicale était de contribuer à une épistémologie de la musique », BERIO, Entretiens avec Rossana Dalmonte, p. 27.

23. Voir FENEYRou Laurent, « Musiques informelles... », p. 735-776.

24. "[I do believe that we must] "think" [our problems] in a Gramscian way ». HALL Stuart, « Gramsci and us », Marxism Today, 1987, p. 16. 
25. Par exemple, Robert Adlington considère que Luigi Nono s'est mépris dans sa lecture de Gramsci : «Considéré habituellement comme un gramscien engagé, Nono a avancé une lecture de Gramsci qui était en fait très idiosyncrasique, résultant en une position qui, à bien des égards, déformait les arguments de son compatriote.» ("Commonly regarded as a committed Gramscian, Nono advanced a reading of Gramsci that was in fact highly idiosyncratic, resulting in a stance that in significant respects misrepresented his compatriot's arguments.") ADLINGTON, « Whose Voices?... », p. 181.

26. Pour un ouvrage abordant les différentes interprétations de l'œuvre de Gramsci en Italie, voir LIGUORI Guido, Gramsci conteso : Interpretazioni, dibattiti e polemiche (1922-2012), Rome, Editori Riuniti, 2012.

27. Palmiro Togliatti (1893-1964) a aussi été membre du Komintern, ministre de la Justice (1945-1946) et membre de l'Assemblée constituante de la République italienne.

28. GRAMSCI Antonio, Lettere dal carcere, sous la direction de Palmiro Togliatti et de Felice Platone, Turin, Einaudi, 1947. Cette édition contient 218 lettres, écrites entre 1926 et 1937.

29. GRAMSCI Antonio, Quaderni del carcere, Turin, Einaudi, 1948-1951. Cette œuvre a été publiée en six volumes: Il materialismo storico e la filosofia di Benedetto Croce [1948], Gli intellettuali e l'organizzazione della cultura [1949], Il Risorgimento [1949], Note sul Machiavelli, sulla politica e sullo Stato moderno [1949], Letteratura e vita nazionale [1950] et Passato e presente [1951]. Concernant le contexte de publication de cette œuvre et ses premières interprétations, voir LIGUORI, Gramsci conteso..., p. 89-132.

30. Signalons notamment l'édition critique de Valentino Gerratana: GRAMSCI Antonio, Quaderni del Carcere, édition critique sous la direction de Valentino Gerratana, 4 vol., Turin, Einaudi, 1975.

31. "Gramsci was "appropriated" by the Party (particularly by its leadership gathered around its secretary Palmiro Togliatti) soon after the end of the war, and his reading (as well as the editorial work around his unpublished works) became a cornerstone in the building of an "imagined continuity" of the history and politics of the party since its foundation in 1921." CAPUZzo Paolo et MEZZADRA Sandro, "Provincializing the Italian Reading of Gramsci ", SRIVASTAVA Neelam et BHATTACHARYA Baidik (dir.), The Postcolonial Gramsci, New York, Routledge, 2012, p. 34.

32. Voir LIGUORI, Gramsci conteso..., p. 121-127. Selon Liguori, il existe deux principales lectures de l'œuvre de Gramsci: la lecture «communiste, togliattienne, antitogliattienne ou post-togliattienne ", d'une part, et la lecture "libérale-démocratique, libérale-socialiste, actionniste ", d'autre part (ibid., p. 17).

33. On peut supposer que Luigi Dallapiccola et Salvatore Quasimodo ont joué un rôle dans cette découverte. Concernant l'influence de Gramsci sur Dallapiccola, voir SAMUEL Jamuna, « Ethics and Musical Language: A Gramscian Reading of Dallapiccola's Liriche greche and Their Influence ", Revue canadienne de musique, XXXV, $\mathrm{n}^{\circ}$ 1, 2015, p. 123-153. À propos de Salvatore Quasimodo (1901-1968), écrivain, poète et prix Nobel de littérature en 1959, nous tenons à rappeler qu'il a aussi été professeur de littérature italienne au Conservatoire de Milan, où Berio et Manzoni ont suivi leurs études en composition. Quasimodo rend hommage à Gramsci dans son « Discours sur la poésie » : "Gramsci voyait clairement, depuis le noir de sa prison, les raisons "littéraires" du monde. La position du poète dans la société ne peut être passive: il "modifie" le monde. » ("Gramsci vedeva con occhi chiari dal buio della sua prigione le ragioni "letterarie" 
del mondo. La posizione del poeta non può essere passiva nella società: egli "modifica" il mondo. ») QUASIMODO Salvatore, "Discorso sulla poesia ", Il Falso e vero verde. Con un discorso sulla poesia, Milan, A. Mondadori, 1956, p. 88.

34. "Proprio in quegli anni lessi una quantità di scritti di Marx, di Lenin, di Engels, di Gramsci che usciva allora da Einaudi. Fu un avvicinarsi progressivo che coincise, qualche anno più avanti, con l'attività di critico musicale a "l'Unità". " MANZONI, « Musica nuova... ", p. 442.

35. " 1945 las ich die Arbeiten des KPI-Gründers Antonio Gramsci. Seit dieser Zeit ist die ideologische Präsenz von Marx mein Ausgangspunkt. Der Kampf gegen den Faschismus und Imperialismus ist mein Lebensinhalt. Ich bin nur zufällig Musiker. » NONO Luigi, «Gesprächt mit Bertram Bock » [1970], Texte, Studien zu seiner Musik, Zurich, Atlantis, 1975, p. 231. La date de 1945 s'explique sans doute par le fait que les écrits de Gramsci avaient commencé à paraître, sous la forme d'extraits, avant la publication des Lettres et des Cahiers, grâce à l'initiative de Togliatti et du quotidien L'Unità (voir LIGUORI, Gramsci conteso..., p. 68-82).

36. " it became, once more, a matter of urgency to form a new culture that could give genuine expression to the national spirit while at the same time featuring the social classes who had previously been excluded as protagonists ». BORIO, "Key Questions... », p. 177.

37. Concernant la vie et l'œuvre d'Ernesto De Martino (1908-1965), voir CHARUTY Giordana, « Être ensemble dans la même histoire : l'œuvre-vie d'Ernesto De Martino », Bérose - Encyclopédie internationale des histoires de l'anthropologie, Paris, 2018 (en ligne : https://www.berose.fr/article1428.html, consulté le 1 mars 2021). Concernant l'influence de De Martino sur la culture musicale italienne de l'après-guerre, voir BORIO, «Key Questions...», p.178-181. Parmi les écrits de De Martino abordant des thématiques gramsciennes, mentionnons «Autour d'une histoire du monde populaire subalterne » (1949), dans lequel l'auteur instaure un parallèle entre les pays du Tiers Monde et les populations subalternes des pays hégémoniques (DE MARTINO Ernesto, «Intorno a una storia del mondo popolare subalterno », Società, 1949, n 3, p. 411-435).

38. Comme le souligne Gianmario Borio, dans la perspective de De Martino « le folklore était considéré comme une partie de l'histoire de la culture dominante, à laquelle il s'opposait cependant; en fixant une "limite" ou un obstacle au déploiement de l'hégémonie, le folklore a immédiatement pris une valeur politique, comme synonyme d'opposition tout court " ( folklore was seen as part of the history of the dominant culture while at the same time standing in opposition to it; by setting a "limit" or obstacle to the deployment of hegemony it immediately took on a political valence, as a synonym for opposition tout court. »), BORIO, « Key Questions... », p. 180.

39. Fondé par Maderna et Berio en 1955, le Studio de phonologie musicale (Studio di fonologia musicale della RAI) fut un laboratoire de renommée internationale, consacré à la recherche et à la création dans le domaine des musiques électroniques.

40. «À Milan, avec Bruno, et indirectement avec Nono, on était en train de former un cercle d'intérêts autour du Studio de Phonologie musicale de la RAI, et autour des concerts et de la revue Incontri Musicali [...]. Rognoni, Leydi, Enzo Paci et Eco étaient nos compagnons de route ", BERIO, Entretiens avec Rossana Dalmonte, p. 45.

41. La philosophie de la praxis est " philosophie de l'histoire, conception du monde exclusivement ancrée dans le réel, pensée de l'immanence, discours pleinement "terrestre" ", HOARE George et SPERBER Nathan, Introduction à Antonio Gramsci, Paris, La Découverte, 2019 [2013], p. 88. 
42. Selon Giovanni Gentile (1875-1944), philosophe idéaliste, puis ministre de l'Éducation au service du régime fasciste, la pensée est un "acte pur ", alors que Gramsci défend une philosophie de l'acte impur, selon laquelle la réalité est toujours le fruit du rapport historique que les hommes entretiennent avec elle. Quant au philosophe et historien Benedetto Croce (1866-1952), il revendique, par la dialectique des distincts, l'autonomie des formes de l'esprit et prend ainsi ses distances avec le marxisme, qui l'a pourtant influencé. Togliatti a qualifié Croce de "pape laïque » et a affirmé que "[sa] dictature sur les cercles intellectuels du siècle dernier couvre et assure la dictature des castes bourgeoises réactionnaires dans l'ordre politique et économique " (" il papa laico [...] la cui dittatura sulla intellettualità dell'ultimo secolo copre e assicura la dittatura delle caste borghesi reazionarie nell'ordine economico e politico»), TOGLIATTI Palmiro, «L'eredità letteraria di Gramsci » [1944], Scritti su Gramsci, Rome, Editori Riuniti, 2001, p. 45.

43. GRAMSCI Antonio, Cahiers de prison, Paris, Gallimard, 1978, vol. III, C11, §30, p. 240.

44. Concernant les concepts de matière et de dialectique chez Nono et chez Manzoni, voir MILLI Pietro, "Dialectics and Matter in the Aesthetics of Giacomo Manzoni", Archival Notes, $\mathrm{n}^{\circ}$ 5, 2020, p. 47-58.

45. NONO Luigi, «Présence historique dans la musique d'aujourd'hui» [1960], Écrits, p. 74-75.

46. C'est à partir de ce constat que Togliatti affirme : «Nous connaissons en opérant. C'est dans l'action pratique et à travers elle que nous nous rendons compte de la structure du monde réel, des rapports économiques, des rapports sociaux et politiques, de l'évolution de leurs lois et tendances, et la connaissance perfectionne à son tour l'action, la rend adéquate au cours des choses» («Noi conosciamo operando. È nell'azione pratica e attraverso di essa che ci rendiamo conto della struttura del mondo reale, dei rapporti economici, dei rapporti sociali e politici, delle loro leggi e tendenze di sviluppo, e la conoscenza a sua volta perfeziona l'azione, la rende adeguata al corso delle cose», TOGLIATti Palmiro, "Storia come pensiero e come azione » [1954], Scritti su Gramsci, p. 191.

47. Concernant la notion d'acte musical, Berio affirme : " Agir musicalement veut dire, pour moi, rendre complémentaires, harmoniser les termes d'une opposition ou d'un ensemble d'oppositions, c'est-à-dire les rendre concrets ", BERIO, Entretiens avec Rossana Dalmonte, p. 102.

48. « die Zusammenarbeit, der gemeinsame Kampf mit den Arbeitern, der ideologische Moment, der revolutionäre Moment und der praktische Moment müssen zusammenfallen. » NONO Luigi, « Gespräch mit Guy Wagner » [1971], Texte, Studien zu seiner Musik, p. 261.

49. À propos du débat autour du rapport entre politique et culture chez Croce et chez Togliatti, voir LIGUORI, Gramsci..., p. 59-68.

50. BERIO Luciano, «Méditation sur un cheval de douze sons » [1968], Entretiens avec Rossana Dalmonte, p. 167-171.

51. Ibid., p. 170-171.

52. «È bene credere nei grandi rivoluzionari, vi sono coloro che sono riusciti ad esserlo tutta la vita, senza passare all'insegnamento di un Conservatorio contro il quale avevano un tempo lanciato $i$ loro calamai, ad esempio Beethoven, Sade, Gramsci sono i morti sulla breccia." BussotTI Sylvano, entretien avec Gioacchino Lanza Tomasi [1965], cité in ESPosito Luigi, Un male incontenibile. Sylvano Bussotti, artista senza confini, Bietti, Milan, 2013, p. 109. 
53. Boulez décrit ainsi la démarche qu'il a adoptée dans cet ouvrage : « Me livrant à une investigation méthodique de l'univers musical, déduisant des conséquences multiples d'un certain nombre de points de départ rationnels, j'ai tenté de construire un système cohérent. » BOUlEz Pierre, Penser la musique aujourd'hui, Paris, Denoël/Gonthier, 1987 [1963], p. 166.

54. FoucAult Michel, «Pierre Boulez ou l'écran traversé », préface posthume à BOULEZ Pierre, Leçons de musique: deux décennies d'enseignement au Collège de France (1976-1995), Paris, Christian Bourgois, 2005 [1982], p. 22.

55. C'est pour cette raison que Norberto Bobbio a fait de Gramsci, à tort ou à raison, le "théoricien des superstructures». Cette lecture oublierait pourtant que les rapports entre superstructure et infrastructure sont dialectiques (voir à ce sujet LIGUORI, Gramsci conteso..., p. 199-203).

56. BERIO, « Méditation sur un cheval de douze sons », p. 168.

57. "Ich halte es mit Antonio Gramsci. Man muss als Komponist die aktuellen technischen Mittel benützen; nicht aus rein ästhetischen Überlegungen, nicht im Dienst abstrakt technologisher Evolution, nicht passiv, sondern aktiv: zur Verbreitung der Ideen, auf die es im Klassenkampf ankommt. Natürlich bilden und fixieren die Produktionsverhältnisse die Voraussetzung für Kunst. Aber auch der Überbau, zu dem der Bereich der Kunst gehört, kann in einer dialektischen Beziehung zur Basis eine Funktion bekommen. Das erleben wir doch heute an den Studentenbewegungen überall auf der Welt: da wird vom Überbau aus die Basis beeinflusst. » NONO Luigi, « Gespräch mit Hansjörg Pauli » [1969], Texte, Studien zu seiner Musik, p. 203.

58. Voir PESTALOzZA Luigi, «Impegno ideologico e tecnologia elettronica nelle opere degli anni Sessanta », RESTAGNO Enzo (dir.), Nono, Turin, EDT, 1987, p. 143-156.

59. Concernant Atomtod de Manzoni, qui aborde ici le thème d'une guerre atomique, voir mILLI Pietro, "Théâtre musical et matiérisme: Atomtod de Giacomo Manzoni ", Itamar, $\mathrm{n}^{0}$ 5, 2019, p. 187-206.

60. Pour une analyse des enjeux politiques de ces œuvres, voir FENEYROU Laurent, « ...la révolution ne renie pas la beauté... Musique et marxisme dans l'Italie d'après 1945 ", FENEYROU Laurent (dir.), Résistances et utopies sonores, p. 149-180.

61. Dans les années 1970, Berio, avec la collaboration de Vittoria Ottolenghi et de Gianfranco Mingozzi, a travaillé pour une série télévisée sur la musique intitulée C’è musica e musica ( Il y a musique et musique »). En évoquant cette expérience dans un entretien avec Rossana Dalmonte, Berio affirme : «Je pense que la fonction principale des mass media, quand on les emploie avec courage, de manière responsable et non selon des critères de rentabilité, est justement de faire évoluer et d'améliorer le goût et les critères de choix des gens ", BERIO, Entretiens avec Rossana Dalmonte, p. 32.

62. "Often, especially in the 1960s and 1970s, at the initiative of musicians or various organizations (above all by parties on the left, societies, or trade unions), concerts were held in unusual venues, in factory buildings, in tents at union rallies, and at the festivals of l'Unità. At such events, "virgin" audiences (so to speak) would flock to hear major international performers, and even works by living composers, and they showed themselves to be sincerely interested and ready to take part; it was a concrete way to make understood to persons excluded from so much of culture how this music constituted a fundamental patrimony, a true and inalienable right for all, whose universal attainment required us all to lead a battle of civilization aimed at a radical transformation of the structures operative in our country», MANZONI Giacomo, "Towards 
Political and Musical Renewal: The Other Idea of Communism », ADLINGTON Robert (dir.), Red Strains: Music and Communism outside the Communist Bloc, Londres, British Academy, 2013, p. 28.

63. Il Diapason, revue du Centro Internazionale di Musica, Milan, 1950-1955.

64. Pour une illustration des différences entre ces deux types d'intellectuels, voir HOARE et SPERBER, Introduction à Antonio Gramsci, p. 29-33.

65. GRAMSCI Antonio, Cahiers de prison, Paris, Gallimard, 1978, vol. III, C12, §3, p. 346.

66. La liste suivante n'est bien évidemment pas exhaustive.

67. "l'essere compositore era uno dei tanti volti, certo il più importante, della sua vita di musicista. [...] La sua partecipazione alla musica è sempre stata quella di un uomo tutto intero $e$ completo. C'era, fra i suoi sensi, le sue emozioni, il suo intelletto, il suo intuito e il suo rigore, una unità molto preziosa e profonda che, in un modo o nell'altro, è sempre stata il tema universale della musica: che è appunto quello di trovare e di perfezionare un'intesa fra dimensioni diverse, fra il dire e il fare, fra il pensare e l'agire, fra "il corpo e l'anima". " BERIO Luciano, « Bruno e la gioia di far musica» [1975], Scritti sulla musica, Turin, Einaudi, 2013, p. 314-315. Le texte dactylographié de cet hommage, rédigé à l'origine en anglais, est conservé à la Paul Sacher Stiftung de Bâle (http://www.lucianoberio.org/omaggio-maderna, consulté le 15 mars 2021).

68. "L'attività multiforme di Schönberg, scrittore, polemista, poeta, pittore, didatta, inventore, teorico, "politico" nel senso alto del termine, ci rimanda all'utopia dell'uomo pienamente realizzato in ogni sua capacità come fu intravista da Karl Marx, se non al concetto di "intellettuale organico" di Antonio Gramsci. » MANzoNI Giacomo, "Il nostro Schönberg" [2011], LOMBARDI Daniele (dir.), Giacomo Manzoni: Pensare attraverso il suono, Milan, Mudima, 2016, p. 376.

69. Voir notamment NONO Luigi, «Le musicien à l'usine » [1966], Écrits, p. 237-240 et MANZONI Giacomo, « Le compositeur et le public » [1990], Écrits, p. 483-485.

70. «La liaison organique modifie les rapports entre les intellectuels, pris au sens large comme dirigeants dans chacun de leurs domaines, et le peuple, créant ainsi une relation dialectique étroite entre dirigeants et dirigés: c'est alors qu'apparaît le nouveau "bloc historique", comme unité entre infrastructure et superstructure dont les intellectuels assurent la liaison. » MACcIoccHI Maria-Antonietta, Pour Gramsci, Paris, Le Seuil, 1974, p. 216. Rappelons que la conception gramscienne de l'intellectuel organique fut critiquée, dans les années 1970, par Alberto Asor Rosa (1933), historien de la littérature ayant fait partie du mouvement opéraïste et auteur de Intellettuali e classe operaia. Selon Asor Rosa, Gramsci a surévalué le rôle des intellectuels, les poussant à considérer le peuple comme un élément amorphe. Voir LIGUoRI, Gramsci conteso..., p. 240-241.

71. «La philosophie de la praxis présuppose tout ce passé culturel, la Renaissance et la Réforme, la philosophie allemande et la Révolution française, le calvinisme et l'économie classique anglaise, le libéralisme laïque et l'historicisme qui est à la base de toute la conception moderne de la vie. La philosophie de la praxis est le couronnement de tout ce mouvement de réforme intellectuelle et morale, dialectisé dans l'opposition culture populaire et haute culture. Elle correspond à la connexion: Réforme protestante + Révolution française : c'est une philosophie qui est aussi une politique, et c'est une politique qui est aussi une philosophie.» GRAMSCI Antonio, Cahiers de prison, Paris, Gallimard, 1990, vol. IV, C16, §9, p. 211. 
72. Maderna cite notamment une chanson en dialecte vénitien intitulée La biondina in gondoleta (La blondinette dans sa petite gondole).

73. Nono cite, entre autres, Non siam più la Comune di Parigi (Nous ne sommes plus la Commune de Paris) et Doubinouchka, chant populaire russe. Dans cette œuvre apparaît également une phrase que Gramsci aurait prononcée lors de son procès: "Vous mènerez l'Italie à la ruine, il reviendra à nous communistes de la sauver » («Voi condurrete l'Italia alla rovina, spetterà a noi comunisti salvarla »).

74. BERIO Luciano, « Folk Songs (note de l'auteur) » (http://www.lucianoberio.org/node/ 1380 ?37697026=1, consulté le 15 mars 2021).

75. BERIO Luciano, « Coro (note de l'auteur) » (http://www.lucianoberio.org/node/1435? 1048623366=1, consulté le 15 mars 2021).

76. Entre 1958 et 1962, ce groupe de musiciens, poètes et écrivains (dont Italo Calvino, Umberto Eco, Franco Fortini), se proposait de renouveler la culture et la musique italienne dans une perspective anticonformiste. Manzoni y a contribué avec une chanson sur un texte anticlérical de Franco Fortini, intitulée Le campane di Roma (« Les cloches de Rome», 1959): "[Elle] n'a jamais été publiée par crainte de rétorsions démesurées de la police et du clergé (mais je souligne que ce n'est pas moi qui me suis retiré) : elle a été rarement interprétée, le plus souvent par Fausto Amodei avec sa guitare - cela a entraîné des dénonciations et des suppressions de spectacles - dans les manifestations que le groupe menait dans l'Italie du centre, pendant les fêtes de L'Unità et d'Avanti! » ("Non fu mai edita per timore di spropositate ritorsioni poliziesche e clericali ( $m a$ devo dichiarare che non fui io a tirarmi indietro): fu di rado eseguita per lo più da Fausto Amodei con la sua chitarra, - in effetti con conseguenti denunce e soppressioni di spettacoli - nelle manifestazioni che il gruppo andava tenendo in mezza Italia durante feste dell'Unità $e$ dell'Avanti! »), MAnzoni Giacomo, Parole per musica, Palerme, L'Epos, 2007, p. 28.

77. La découverte de chants populaires siciliens par les compositeurs en question a été rendue possible notamment par la publication de cet ouvrage : FAVARA Antonio, Corpus di musiche popolari siciliane, Palerme, Accademia di scienza, lettere e arti, 1957. Concernant l'intérêt de Berio pour les musiques populaires, voir BERIO, Entretiens avec Rossana Dalmonte, p. 81-82.

78. GRAMSCI Antonio, Cahiers de prison, Paris, Gallimard, 1992, vol. V, C29, §3, p. 369.

79. La « guerre de positions » désigne " une "guerre de siège" sur le terrain des luttes culturelles et idéologiques au sein de la société civile », HOARE et SPERBER, Introduction à Antonio Gramsci, p. 50.

80. Voir NONO, « Le pouvoir musical », Écrits, p. 312-323.

81. Voir Pestalozza, « Gramsci... », p. 35.

82. Par exemple Al gran sole carico d'amore (1974), où Nono aborde le thème de la révolution en utilisant des textes en différentes langues (italien, français, espagnol, allemand...).

83. Citons Quattro canzoni popolari (1952) de Berio, pour voix de femme et piano et sur des textes anonymes en sicilien et en génois, ainsi que sur un poème de Jacopo da Lentini. Son intérêt pour la musique et les textes siciliens se poursuit jusqu'aux années 2000, avec E si fussi pisci (2002), pour chœur. Quant à Manzoni, il a mis en musique des textes en sicilien (Cinque vicariote, 1958, pour chœur et orchestre), en frioulan (Uéi preà la biele stele 1987, pour chœur d'hommes à l'unisson et une ou deux grosse(s) caisse(s)), 
et en véronais (Canzonetta "Se mi fusse", 1996, pour dix voix de femme, crotales, cymbales suspendues et tambour).

84. NONO Luigi, «Face au conflit historique de l'époque, le compositeur doit adopter un comportement responsable. Entretien avec José Antonio Alcaraz » [1964], Écrits, p. 182.

85. Idem.

86. MANZONI Giacomo, «Le long chemin du Docteur Faustus » [1987], Écrits, Paris, Basalte, 2006, p. 282.

87. Voir PESTALOZZA Luigi, «Stockhausen et l'autoritarisme musical " [1968], Musique, rupture, p. 55-67.

88. NONO, « Le pouvoir musical », p. 317.

89. NONO, « Entretien avec Luigi Nono » [1969], Écrits, p. 298.

90. GRAMSCI Antonio, Cahiers de prison, Paris, Gallimard, 1983, vol. II, C9, §66, p. 445.

91. Ibid., p. 446.

92. Idem.

93. "Di grande importanza è stata inoltre la riscoperta del melodramma italiano, dopo la caduta del fascismo, alla luce delle letture di Gramsci e di Lukács, alla luce cioè della critica marxista. ", BERIO Luciano, «Verdi? » [1974], Scritti sulla musica, p. 123.

94. Dans les indications de mise en scène de cette œuvre, Bussotti recommande de s'inspirer du mobilier et d'objets appartenant au répertoire opératique italien, comme le divan de Traviata, le lit d'Otello ou l'agenouilloir de Don Carlos.

95. Voir BERIO, «Verdi? », p. 121-127 et MANZONI Giacomo, "Tra "suono" e "rumore" in Otello e Lulu » [2001], Musica e progetto civile, p. 372-376. Ce dernier instaure un parallèle entre Lulu d'Alban Berg et Otello de Verdi, dont il souligne les nouveautés d'un point de vue harmonique et timbrique.

96. NOLLER, « Gramsci e la musica », p. 44.

97. Par exemple, les références à certains communistes considérés comme non orthodoxes (Bordiga, Trotsky, Rosa Luxemburg...) furent supprimées. Voir CAPUzzo et MEZZADRA, « Provincializing the Italian Reading of Gramsci », p. 36.

98. "These letters highlighted the human side of Gramsci and they assumed a paradigmatic meaning of the violence suffered by all those who had the courage to vigorously oppose the Fascist regime », CAPUZZo et MEZZADRA, "Provincializing the Italian Reading of Gramsci », p. 36.

99. PESTAlozZA, « Gramsci... », p. 37.

100. Ibid., p. 38.

101. Pour une analyse de cette œuvre et de son rapport avec la pensée de Gramsci, voir VERZINA Nicola, «Tecnica della mutazione e tecnica seriale in Vier Briefe (1953) di Bruno Maderna », Rivista italiana di musicologia, XXXIV, nº 2, 1999, p. 309-346.

102. MILA Massimo, «Un omaggio musicale all'opera di Gramsci », La Stampa, 28 août 1974.

103. BussotTi Sylvano, extrait d'une lettre à Giacomo Manzoni, datant du $1^{\mathrm{er}}$ septembre 1977, cité in MANZONI, Écrits, p. 232.

104. Selon Asor Rosa, les positions de Gramsci étaient acceptées "parce que cette reconnaissance leur permettait d'affirmer leur hégémonie dans le cadre du bloc 
historique et même à l'intérieur du parti. » (" perché tale riconoscimento permetteva loro di affermare la propria egemonia nell'ambito del blocco storico e persino dentro il partito. ») ASOR ROSA Alberto, Intellettuali e classe operaia, Florence, La Nuova Italia, 1973, p. 572.

105. "We aspired to a new society in Italy, distinct from the USSR, more attentive to human rights, to new ideas regarding development, research and freedom, in music and in the arts in general. » MANZONI, « Towards Political and Musical Renewal... », p. 24.

106. «La tradizione italiana e il metodo di Gramsci divenivano [...] la ricerca di un terreno d'incontro con la cultura del nostro paese; ma erano anche il modo e il mezzo [...] per prendere le distanze dal modello staliniano-zdanovista senza al contempo determinare una rottura aperta, politicamente insostenibile », LIGUORI, Gramsci conteso..., p. 96.

107. LosuRDo Domenico, «Avec Gramsci, par-delà Marx et par-delà Gramsci », Nouvelles Fondations, 2007/3-4, $\mathrm{n}^{\circ}$ 7-8, p. 210.

108. ADORNo Theodor W., Théorie esthétique, Paris, Klincksieck, 1974 [1970].

109. MANZoNi Giacomo, «Contre une "esthétique" de la musique aujourd'hui » [1995], Écrits, p. 246.

\section{RÉSUMÉS}

Cette étude aborde l'influence de l'œuvre de Gramsci sur cinq compositeurs italiens d'aprèsguerre: Bruno Maderna, Luigi Nono, Luciano Berio, Sylvano Bussotti et Giacomo Manzoni. En nous appuyant sur leurs écrits, sur leurs œuvres et sur leurs parcours militants et artistiques, nous chercherons à montrer en quoi la pensée gramscienne a pénétré leurs discours et leurs pratiques. Pour ce faire, nous étudierons dans un premier temps des questions de nature philosophique et sociologique (le rapport entre théorie et praxis, superstructure et infrastructure...), puis des problématiques d'ordre linguistique (la «question de la langue », la dialectique cosmopolitisme/national-populaire...). Nous avancerons enfin quelques observations concernant l'utilisation musicale et politique de l'œuvre de Gramsci et son rôle au sein de la culture italienne, notamment en rapport à la pensée d'Adorno.

This study addresses the influence of Gramsci's work on five Italian composers of the second post-war period: Bruno Maderna, Luigi Nono, Luciano Berio, Sylvano Bussotti and Giacomo Manzoni. Based on their writings, their works and their activist and artistic careers, we will try to show how Gramscian thought has penetrated their discourses and practices. To this end, we will first study philosophical and sociological questions (the relationship between theory and praxis, superstructure and infrastructure, etc.), then linguistic issues (the "language question", the dialectic of cosmopolitanism/national-popular, etc.). Finally, we will make some observations concerning the musical and political use of Gramsci's work and its role in Italian culture, especially in relation to Adorno's thought. 
INDEX

Keywords : Antonio Gramsci, 20th century music, Bruno Maderna, Luigi Nono, Luciano Berio, Sylvano Bussotti, Giacomo Manzoni

Mots-clés : Antonio Gramsci, musique du xxe siècle, Bruno Maderna, Luigi Nono, Luciano Berio, Sylvano Bussotti, Giacomo Manzoni

\section{AUTEUR}

\section{PIETRO MILLI}

Pietro Milli est chercheur associé au Groupe de Recherche d'Histoire (laboratoire GRHis, Université de Rouen). Après des études en musicologie à l'Université Ca' Foscari de Venise, à la Sorbonne et à l'EHESS, il a soutenu une thèse sur l'œuvre et la poétique de Giacomo Manzoni sous la direction de Pierre-Albert Castanet et de Laurent Feneyrou. Ses recherches portent notamment sur la musique italienne de l'après-guerre et sur les écrits de compositeurs. Il contribue régulièrement à des revues et à des projets en ligne (Lectures, Dictéco, Itamar...), ainsi qu'à des ouvrages collectifs. 